\title{
Towards adaptive mesh refinement for the spectral element solver Nek5000
}

\author{
N. Offermans, A. Peplinski, O. Marin, P. F. Fischer, and P. Schlatter
}

\section{Introduction}

When performing computational fluid dynamics (CFD) simulations of complex flows, the a priori knowledge of the flow physics and the location of the dominant flow features are usually unknown. For this reason, the development of adaptive remeshing techniques is crucial for large-scale computational problems. Some work has been made recently to provide Nek5000 with adaptive mesh refinement (AMR) capabilities in order to facilitate the generation of the grid and push forward the limit in terms of problem size and complexity. Nek5000 is an open-source, highly scalable and portable code based on the spectral element method (SEM), which offers minimal dissipation and dispersion, high accuracy and nearly exponential convergence. It is aimed at solving direct numerical simulations of turbulent incompressible or low Mach-number flows with heat transfer and species transport. The approach chosen for adapting the mesh is the h-refinement method, where elements are split locally, which requires the relaxation of the conforming grid constraint currently imposed by Nek5000. Other challenges include the implementation of an efficient management of the grid as refinement is applied, the development of tools to localize the critical flow regions via error estimators and the extension of the current preconditioning strategy to non-conforming grids. In this paper, we present a new procedure to setup an algebraic multigrid solver used as part of the preconditioner for the pressure equation.

N. Offermans $\cdot$ A. Peplinski $\cdot$ P. Schlatter

Linné FLOW Centre, KTH Mechanics, Royal Institute of Technology, Sweden, e-mail: \{nof, adam, pschlatt\}@kth. mech.se

O. Marin

Mathematics and Computer Science Division, Argonne National Laboratory, USA, e-mail: oanamemcs.anl.gov

P. F. Fischer

Siebel Center for Computer Science, University of Illinois, Urbana-Champaign, USA, e-mail: fischerp@illinois.edu 


\section{Pressure preconditioning in Nek5000}

\subsection{Coarse grid solver}

The major source of stiffness when solving the Navier-Stokes equations comes from the pressure equation, which requires an efficient preconditioning strategy. The method chosen for Nek5000 is called additive Schwarz [1] and the preconditioner can be expressed as

$$
M_{0}^{-1}=R_{0}^{T} A_{0}^{-1} R_{0}+\sum_{k=1}^{K} R_{k}^{T} A_{k}^{-1} R_{k},
$$

where $K$ is the number of spectral elements and $R_{k}$ and $R_{0}$ are restrictions operators. This preconditioner can be seen as the sum of the global coarse grid operator (subscript 0) and local subdomain operators (subscript k). The present work focuses on the solution of the coarse grid operator, $A_{0}$, which is constructed as a finite element Laplacian matrix. The so-called "coarse grid" denotes the grid defined by the user. At the beginning of each simulation, the final mesh is built by filling each of the elements of this grid by inner collocation points, whose number depends on the chosen polynomial order for the spectral expansion. The coarse grid problem does not take these inner collocation points into account. Two choices are available in Nek5000 to solve this problem. The first one is a direct, sparse basis projection method, called XXT [2]. The second option uses an algebraic multigrid (AMG) method, which is more efficient for massively parallel (more thand 10,000 cores) large simulations (more than 100,000 elements) [3]. As usual with AMG methods, a setup step is required for the matrix $A_{0}$, which will define the necessary data for solving the problem: a coarsening operation defines the different levels of the multigrid, the interpolation operators between the levels are computed and a smoother is computed at each level. In the particular case of Nek5000, the AMG solver performs a single $\mathrm{V}$-cycle and a fixed number of Chebyshev iterations, computed during the setup, is applied during the smoothing part. This method has the big advantage to avoid inner product, thus reducing communication, at the expense of requiring some knowledge about the eigenvalues of the operator. More information about the theoretical background for the setup can be found in [4]. While the AMG solver is highly scalable and efficient, the setup phase is currently performed by a serial Matlab code, which can take up to a few hours for the largest current cases on a modern desktop computer. This bottleneck is an obstacle to the use of AMR, where the grid, therefore the operator $A_{0}$, is modified regularly, every time requiring a new setup computation. For that reason, a new method has been investigated to replace the Matlab setup. 


\subsection{Use of Hypre for the AMG setup}

As an alternative way of performing the setup, the Hypre library for linear algebra is used [5]. Specifically, only the time consuming coarsening and interpolation operations are performed with Hypre, while the computation of the smoother operators remains unchanged in order to keep the good performance of the AMG solver. The use of Hypre offers the possibility to choose among various algorithms for coarsening and interpolation. For the current tests, we chose the Ruge-Stueben algorithm for the coarsening and the so-called "classical" modified technique for the interpolation. Furthermore, an input parameter allows the user to specify a target tolerance for the convergence factor of the smoother. This parameter directly impacts the number of Chebyshev iterations at each level and enables the user to set a compromise between accuracy and rapidity of the AMG solver. Despite the parallel capabilities of Hypre, the setup is performed by a serial, external $\mathrm{C}$ code. The main goal with this new setup is to demonstrate two points. First, we want to show that the use of the Hypre library reduces significantly the time required to perform the setup. Then, we want to show that the coarsening and interpolation techniques of Hypre do not affect the efficiency of the AMG solver during the simulations. Additionally, we also wish to check that the XXT solver becomes slow for large cases.

\section{Validation of the Hypre setup}

In this section, we experiment the new setup code on several test cases. In particular, we verify that the Hypre setup is faster than the Matlab one, while the computational time is not affected. We also show the advantage of AMG over XXT for big simulations.

\subsection{Test cases}

The test cases considered are simulations of a jet in a crossflow, of a turbulent straight pipe $\left(R e_{\tau}=550\right)$ and of the flow around a NACA4412 airfoil $(R e=$ $400,000)$. Some basic information about the cases is summarized in table 1. All these cases are flagship simulations, requiring substantial computational resources and are relevant because of their large size and mesh complexity.

\subsection{Timing of the AMG setup}

The timings for the setup are reported in table 2 for the Matlab and the Hypre setups. The total setup time is split between I/O time (reading and writing the setup data) 
Table 1 Summary of the cases used for testing the Hypre setup.

\begin{tabular}{lll}
\hline Case name & Number of elements & Polynomial order \\
\hline Jet in crossflow & 47,960 & 7 \\
Straight pipe & 853,632 & 7 \\
NACA4412 & $1,847,664$ & 11 \\
\hline
\end{tabular}

Table 2 Comparison between the timings of the Matlab and Hypre codes for the AMG setup.

\begin{tabular}{lllll}
\hline & Time (s.) & I/O & Computation & Total \\
\hline Jet in crossflow & Matlab & 1.17 & 97.47 & $\mathbf{9 8 . 6 4}$ \\
& Hypre & 0.4 & 2.02 & $\mathbf{2 . 4 2}$ \\
\hline Straight pipe & Matlab & 26.49 & 2204.55 & $\mathbf{2 2 3 1 . 0 4}$ \\
& Hypre & 32.75 & 44.4 & $\mathbf{7 7 . 1 5}$ \\
\hline NACA4412 & Matlab & 93.98 & 3661.94 & $\mathbf{3 7 5 5 . 9 2}$ \\
& Hypre & 90.4 & 91.7 & $\mathbf{1 8 2 . 1}$ \\
\hline
\end{tabular}

and computational time (coarsening and computation of the interpolation operator and smoother at each level). All setups are performed a single time on the same desktop machine (CPU: Intel Core I7 990 Extreme, RAM: 24gb), in serial.

In all cases, the computational and total times for the setup are reduced by more than one order of magnitude. The timings for I/O remain similar on the other hand. Overall, this provides the proof that Hypre can be used to drastically reduce the setup time.

\subsection{Timing of the simulation}

The mean total wall clock time per time step during the simulation is presented in figures 1 and 2 for the jet in crossflow and the straight pipe respectively. Similarly, the time for the coarse grid solver and the total computational time are shown, per time step, in figures $3 \mathrm{a}$ and $3 \mathrm{~b}$ for the NACA4412 airfoil. For all cases, the reported times correspond to averages over 20 time steps and exclude I/O. Each simulation has been run, once, on Beskow, a Cray XC40 supercomputer based at The Royal Institute of Technology in Stockholm. For the jet in crossflow and the pipe, several number of cores have been considered and the plots show the strong scaling of the code.

The results for the jet in crossflow, illustrated in figure 1, show that the Hypre setup does not affect the time to solution, as both setups are very similar in terms of computational time. Regarding the comparison between AMG and XXT, the simulation of the jet in crossflow is too small to see a consistant gap between both coarse 


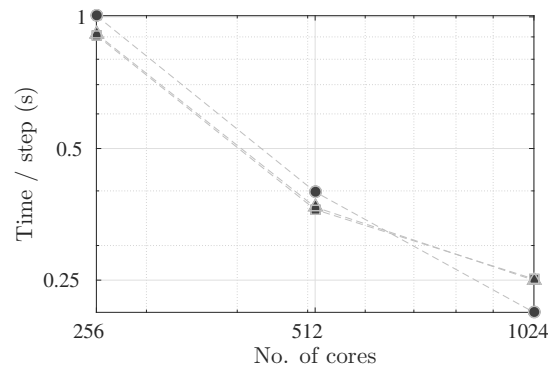

Fig. 1 Figure : Jet in crossflow - Total computational time using XXT (circle), AMG with the Matlab setup (square) and AMG with the Hypre setup (triangle).

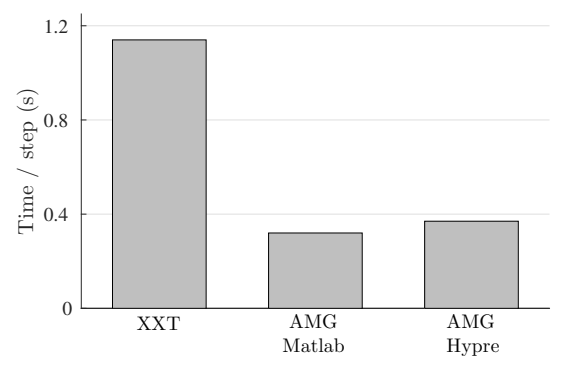

Fig. 3a : NACA4412 airfoil - Coarse grid solver time using XXT, AMG with the Matlab setup and AMG with the Hypre setup.

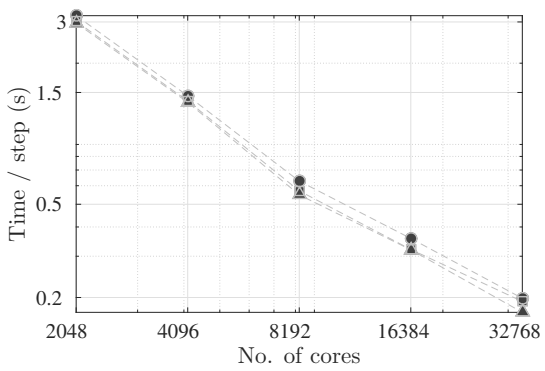

Fig. 2 Figure : Straight pipe $\left(R e_{\tau}=550\right)$ Total computational time using XXT (circle), AMG with the Matlab setup (square) and AMG with the Hypre setup (triangle).

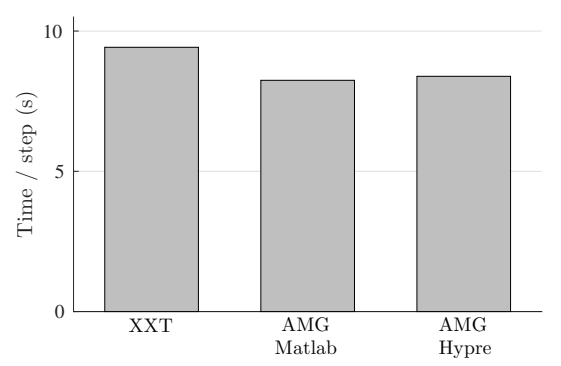

Fig. 3b : NACA4412 airfoil - Total computational time using XXT, AMG with the Matlab setup and AMG with the Hypre setup.

grid solvers, as can be seen by the fact that the best performing method depends on the number of processes.

It is observed again in figure 2 that both the Hypre and the Matlab setups perform similary well in the case of the simulation of a straight pipe. Given the larger size of the case, it also appears that the use of XXT for preconditioning the pressure equation id systematically slower compared to AMG. This difference is a only a few percents but occurs at all numbers of cores considered.

Finally, figures $3 \mathrm{a}$ and $3 \mathrm{~b}$ show once more that either setup method can be used without affecting the solver time. The slightly higher timer for the coarse grid solver may be partly attributed to a higher number of Chebyshev iterations when using Hypre compared to Matlab (33 vs. 26). Other factors that might explain the difference are the different algorithms used for coarsening and interpolation. Both figures also illustrate that the use of AMG should be preferred ovec XXT for large simulations. In the case of the wing, the gain in coarse grid solver time is about $70 \%$, which translates into a reduction of the total computational time by about $10 \%$. 


\section{Conclusion and outlook}

First, with the help of Hypre, the setup time was reduced by more than one order of magnitude compared to the Matlab code. This improvemement will benefit the users of Nek5000 and should facilitate the use of the AMG solver within the framework of adaptive mesh refinement.

Then, it has been shown that the use of AMG instead of XXT for solving the coarse grid problem in Nek5000 significantly improves the time to solution for large cases (typically more than 100,000 elements on more than 10,000 cores).

Finally, it has been observed that replacing the coarsening and interpolation operations of the original Matlab code for the setup by the Hypre routines, while keeping the same strategy for the smoother, does not affect significantly the total time to solution.

In the future, the setup code using Hypre will be parallelized and included inside Nek5000 such that no interuption in the worflow of a simulation is required. The effect of parallelization on the quality of the coarsening and interpolation operations will also be studied.

\section{References}

1. P.F. Fischer: An overlapping Schwarz method for spectral element solution of the incompressible Navier-Stokes equations. J. of Comp. Phys. (1997)

2. H. M. Tufo, P. F. Fischer: Fast Parallel Direct Solvers for Coarse Grid Problems. Journal of Parallel and Distributed Computing 61, number 2, 151-177 (2001) doi: http://dx.doi.org/10.1006/jpdc.2000.1676

3. P. F. Fischer, J. Lottes, D. Pointer, A. Siegel: Petascale algorithms for reactor hydrodynamics, Journal of Physics: Conference Series 125, number 1 (2008)

4. J. Lottes: Towards Robust Algebraic Multigrid Methods for Nonsymmetric Problems, Springer Theses (2017), ISBN: 978-3-319-56305-3 (Print) 978-3-319-56306-0 (Online)

5. Hypre, high performance preconditioners - User's Manual, Center for Applied Scientific Computing Lawrence Livermore National Laboratory 